\title{
IMPROVEMENTS IN THE CR39 POLYMER FOR THE MACRO EXPERIMENT AT THE GRAN SASSO LABORATORY
}

\author{
Presented by $L$. Patrizii for the MACRO collaboration
}

S.P.Ahlen(3), M.Ambrosio(12), G.Auriemma(14), C.G.Babarino(12), A.Baldini(13), B.Barish(4), G.Battistoni(6), R.Bellotti(1), C.Bemporad(13), P.Bernardini(10), H.Bilokon(6), V.Bisi(16), C.Bloise (6), C.Bower(8), S.Bussino(14), F.Cafagna(1), M.Calicchio(1), P.Campana (6), A.Castelvetri(2), S.Cecchini(2), F.Cei(13), V.Chiarella(6), P.Chrysicopoulou(14), R.Cormack (3), S.Coutu(4), G.De Cataldo(1), C.De

Marzo(1), E.M.De Vincenzi(14), E.Diehl(11), E.Erriquez(1), C.Favuzzi(1), D.Ficenec(3), C.Forti( ${ }^{(6)}$, G.Giacomelli(2), G.Giannini(13), N.Giglietto(1), P.Giubellino(16), M.Grassi(13), P.Green(15), A.Grillo(6), F.Guarino(12), C.Gustavino(7), E.Hazen(3), R.Heinz(8), J.T.Hong(4), E.Iarocci(6),

S.Klein (3),E.Lamanna (14), C.Lane(5), D.Levin(3), P.Lipari(14), G.Liu(4), M.Longo(11), G.Mancarella(10), G.Mandrioli(2), A.Margiotta-Neri(2), A.Marin (3), A.Marini(6), G.Martellotti(14), A.MarzariChiesa(16),M.Masera(16), P.Matteuzzi(2), L.Miller(8), P.Monacelli(9),

M.Monteno(16),S.Mulfson(8), J.Musser(11), E.Nappi(1), G.Osteria(12), B.Pal(2), O.Palamara(10), M.Pasquale(16), V.Patera (6), L.Patrizii(2), R.Pazzi(13), C.Peck(4), J.Petrakis(8), S.Petrera(10),N.Pignatano(4), P.Pistilli(10), F.Predieri(2), L.Ramello(10). A.Reale(9), J.Reynoldson(7),F.Ronga(6), G.Rosa (14), G.L.Sanzani(2), C.Satriano(14), L.Satta(6),

E.Scapparone(2),A.Sciubba(14), P.Serra Lugaresi(2), M.Severi(14), G.P.Sini (2), C.Smith(11), D.Solie(4), P.Spinelli(1), M.Spinetti(6), M.Spurio(2), J.Steele (4), R.Steinberg(5), J.L.Stone(3), L.R.Sulak(3), A.Surdo(10), G.Tarle'(11), V.Togo(2), V.Valente(6), R.Webb(15), W.Worstell(3),

1. Dipartimento di Fisica, Universita' di Bari and INFN, Bari, 70126, Italy.

2. Dipartimento di Fisica, Universita' di Bologna and INFN, Bologna, 40126, Italy.

3. Physics Department, Boston University, Boston, MA 02215, USA.

4. California Institute of Technology, Pasadena, CA 91125, USA.

5. Department of Physics, Drexel University. Philadelphia, PA 19104, USA.

6. Laboratori Nazionali di Frascati dell'INFN, Frascati (Roma) 00044, Italy.

7. Laboratori Nazionali del Gran Sasso dell'INFN, Assergi (L'Aquila) 67010 Italy

8. Dept. of Physics, Dept. of Astronomy, Indiana University, Bloomington, IN 47405, USA.

9. Dipartimento di Fisica. Universita' dell'Aquila and INFN, L'Aquila, 67100. Italy.

10. Dipartimento di Fisica, Universita' di Lecee and INFN, Lecce, 73100, Italy.

11. Department of Physics, University of Michigan, Ann Arbor, MI 48109, USA.

12. Dipartimento di Fisica, Universita' di Napoli and INFN, Napoli, 80125, Italy.

13. Dipartimento di Fisica, Universita' di Pisa and INFN, Pisa, 56010. Italy.

14. Dipartimento di Fisica, Universita' di Roma and INFN, Roma, 00185, Italy.

15. Department of Physics, Texas A \& M University, College Station, TX 77843, USA.

16. Dipartimento di Fisica, Universita' di Torino and INFN, Torino, 10125, Italy.

\begin{abstract}
We discuss the preparation of different samples of the CR39 polymer and a number of tests performed with high energy heavy ions. The quality and reproducibility of the manufactured CR39 is discussed as well as the implications for a large area detector (MACRO) used for a search for cosmic magnetic monopoles.
\end{abstract}

\section{KEYWORDS}

CR39 track-etch detector, MACRO experiment, magnetic monopoles, calibration, heavy ions, threshold, ageing. 


\section{THE MACRO TRACK-ETCH DETECTOR}

MACRO is a large area multi-purpose detector, installed in the Hall B of the Gran Sasso National Laboratory. It is designed to investigate the penetrating cosmic ray radiation. Its acceptance for an isotropic particle flux will be about $10000 \mathrm{~m}^{2}$ sr. The detector uses three complementary techniques: liquid scintillators, limited streamer tubes and track-etch detectors. The MACRO detector (MACRO Collaboration, 1986) is organized in 6 supermodules, each one of $12 \mathrm{~m} \times 12 \mathrm{~m}$ in size and $5 \mathrm{~m}$ height. At present three supermodules are running.

The main purpose of the MACRO track-etch detector (which will have an ultimate size of morc than $1000 \mathrm{~m}^{2}$ ) is the search for magnetic monopoles. It is placed horizontally in the middle of the MACRO structure ; in the future a vertical wall will be added on one side of MACRO. The track-etch detector is organized in 48 "trains" per supermodule; each train has 47 "wagons" of $25 \times 25 \mathrm{~cm}^{2}$ in size. A "wagon" contains three layers of CR39, $1.4 \mathrm{~mm}$ thick, a $1 \mathrm{~mm}$ thick aluminium absorber and three layers of lexan, $0.2 \mathrm{~mm}$ thick. These layers are placed in an aluminium bag filled with dry air. Each "wagon" can be inserted or removed by sliding it on rails.

The passage of a magnetic monopole is expected to cause structural damage in the CR39. Chemical etching should result in collinear etch pits of equal size on both faces of the sheets. The tracketch detector will be used mainly for confirmation, when "triggered" by a signal in the scintillation counters and/or the limited streamer tubes. For these candidate events, a search is performed for a monopole track in the CR39 around the expected position, defined to within few square centimeters. The interesting "wagon" is extracted and one layer of CR39 is heavily etched. A scan for holes is performed with low magnification optical methods. The other two sheets of CR39 are used for confirmation of any candidate monopole track in the heavily etched layer. The relative position of the monopole tracks in the different layers of CR39 can be determined with a precision of better than 100 microns. The technique has already been tested, and proved viable.

\section{THE PRODUCTION OF CR39}

In collaboration with the INTERCAST Company of Parma, Italy, manufacturer of CR39 mainly for sun glasses, we improved the quality of the polymer in order to achieve:

- low detection threshold.

- high sensitivity in a large range of energy losses,

- high quality of the post-etched surface after prolonged etching,

- stability of the sensitivity of the polymer over long periods of time ( several years),

- uniformity of sensitivity for mass-produced sheets.

After a number of tests (MACRO Collaboration, 1988) a first batch of 1000 CR39 sheets, each $98 \times 98$ $\mathrm{cm}^{2}$ in size, was made by INTERCAST; it was used to cover $1 / 4$ of the MACRO surface. The next step was the preparation of new samples of higher quality and higher reproducibility. Major improvements came from the use of a different catalyzer, a different curing cycle and a number of extra precautions. Following these tests, a second and a third batch, each of 1000 sheets, was made by INTERCAST in a new special industrial line, specifically set up for the production of CR39 to be used for track detectors. About 5/6 of the total MACRO area has been already covered. A new production will start in the near future to complete the installation of the track-etch detector. The present discussion will be mainly restricted to two types of CR39: EN3 (first batch) and L6 (second and third batches) that are already installed in MACRO.

\section{BULK ETCHING RATES}

Several samples of CR39, types EN3, L6 and L8, of different chemical composition and made with different curing cycles (Tab.1) were etched in a solution $6 \mathrm{~N}$ of $\mathrm{NaOH}$ at $70^{\circ} \mathrm{C}$ for successive etching times of $5,10,15,20$, and 40 hours. The thicknesses of the sheets were measured after each etching period. Generally the thicknesses increase in the first 5 hours of etching; then they decrease regularly (Fig.1). The slope of the decrease is the bulk etching rate. The increase in thickness at small etching times is connected with a swelling of the plastic, due to the combined effect of water and temperature. The bulk etching rates, $v_{B}$,were determined by fitting the measured data after the first 10 hours of etching. The results are given in Table 1; errors include statistical and systematic uncertainties. The EN3 type has the largest $v_{B}$ value. The post-ctching surface quality and transparency decrease from L8 to L6 and to EN3. This suggested the convenience of the use of the IPP catalyzer instead of the CHPC catalyzer. After a number of tests, we limited the mass production to the L6 type. 
Table1-Chemical composition of different types of CR39, the maximum temperatures reached in the curing cycle and the measured bulk etching rates.

\begin{tabular}{|c|c|c|c|c|c|}
\hline Type & Date & Catalyzer & Additives & $\begin{array}{l}\text { Max. terrp. in } \\
\text { the curing cycle. }\end{array}$ & $V_{B}(\mu m / h)$ \\
\hline ENB & $\operatorname{Jan} .88$ & CHPC & DOP & $80^{\circ} \mathrm{C}$ & $1.21 \pm 0.02$ \\
\hline 16 & $\begin{array}{l}\text { Jul.88, Mar.89, } \\
\text { Jan.90 }\end{array}$ & IPP & $\begin{array}{l}\text { Naugard } \\
\text { antioxydant }\end{array}$ & $95^{\circ} \mathrm{C}$ & $1.16 \pm 0.02$ \\
\hline LB & Jul.88 & IPP & Naugard,DOP & $95^{\circ} \mathrm{C}$ & $1.16 \pm 0.02$ \\
\hline
\end{tabular}

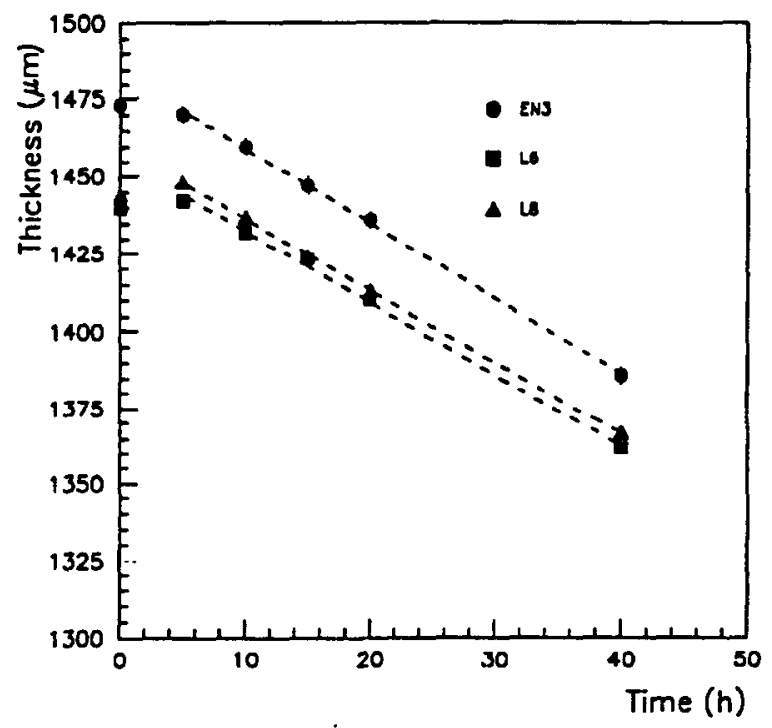

Fig.1. Thickness versus etching time of CR39, types EN3, L6 and L8 . (Etching conditions : $\mathrm{NaOH}, 6 \mathrm{~N}, 70^{\circ} \mathrm{C}$ )

\section{CALIBRATION CURVES}

In order to determine the calibration curves, stacks of CR39 of type EN3 and L6 were exposed to neon ions of $585 \mathrm{MeV} /$ nucleon at the Berkeley Bevalac. The EN3 and L6 samples were 1.5 and 0.5 years old respectively. After production they had been stored in aluminium bags filled with dry air in the Gran Sasso underground laboratory. A stack was typically made of 6 CR39 sheets, a copper absorber $1.4 \mathrm{~cm}$ thick, followed by 10 sheets of CR39. All the CR39 sheets were $13 \times 7 \mathrm{~cm}^{2}$ i n size, and $1.4 \mathrm{~mm}$ thick. The CR39 sheets located after the absorber detected also lower $Z$ fragments from the original ions. After $45 \mathrm{~h}$ of etching in $\mathrm{NaOH} 6 \mathrm{~N}$ at $70^{\circ} \mathrm{C}$, the surface areas of the etch-pits were measured with an Elbek image analyser. From these measurements and the measurement of $V_{B}$ the reduced etch ratio $p=V_{T} / V_{B}$ was calculated for each detected charged fragment using the usual formulae (Fleischer et al., 1976). The obtained values of p are plotted versus the Restricted Energy Loss (REL) in Fig.2 for both EN3 and L6 types. The dashed lines in Fig. 2 are the result of a best fit to a second order polynomial; the extrapolation to $p=1$ of each curve yields the threshold values, $R E L=27$ and $53 \mathrm{MeV} \mathrm{cm} 2 / g$ for $\mathrm{L} 6$ and EN3 types, respectively; these values correspond to $Z_{\text {eff }} / B=5.1$ and 7.3 . The lower threshold and the higher sensitivity of the type L6 is mainly due to 
the absence of the DOP in the chemical composition. The EN3 type should be sensitive to $g=1 g_{D}$ magnetic monopoles with $\beta>2 \times 10^{-3}$ and also for $3 \times 10^{-5}<\beta<1.5 \times 10^{-4}$ if nuclear energy losses can be detected in CR39. The $\beta$-range covered by the L6 type is only slightly larger. We plan to study experimentally the response of CR39 in the very low B-region.

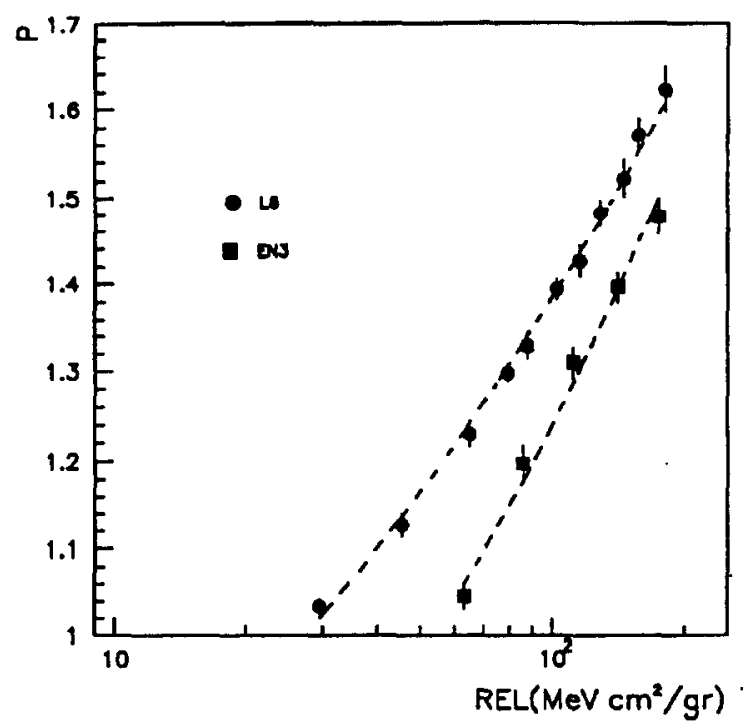

Fig.2. Measured values of the reduced etch-ratio $p=v_{T} / v_{B}$ versus $R E L$ for EN3 and L6 types.

\section{UNIFORMITY OF SENSITIVITY}

Six samples of the L6 type, were laser cut from 3 different sheets of the same batch. One single batch in the INTERCAST production line includes 48 sheets of $98 \times 98 \mathrm{~cm}^{2}$ in size. The samples were exposed in a stack to neon ions of $585 \mathrm{MeV} /$ nucleon, and etched together. The obtained p-values for each sheet, shown in Fig.3, indicate a good uniformity of sensitivity for sheets of the same batch. The improvement in uniformity may depend on the longer stirring time of the mixture as well as on the higher temperatures reached during the curing cycle.

\section{TIME STABILITY}

The Naugard-445 anti-oxydant has been introduced in the $L 6$ type in order to improve the stability of the sensitivity over long periods of time (Doke et al., 1988). Fig.4 shows the experimental calibration points of two sheets of different ages: L6 200 was made 1 year before the exposure and stored in the MACRO environment, while the other, L6 400, was newly made. Four samples were laser cut from each original sheet, and exposed in stacks to $585 \mathrm{MeV} /$ nucleon neon ions. The points in the graph are the averages over 4 samples. The sensitivity to neon ions and to their fragments is the same for both, thus showing the stability of the L6 plastic over 1 year of storage in the MACRO environment; it also suggests the reproducibility of the sensitivity of this type of CR39 in different production batches. We also checked that the CR39 of EN3 type shows no significant loss of sensitivity near the threshold after 1.5 years of installation in the MACRO detector. The result suggests that the sealing of the CR39 in dried air filled aluminium bags at $18^{\circ} \mathrm{C}$ (MACRO environment) are good storage conditions. 


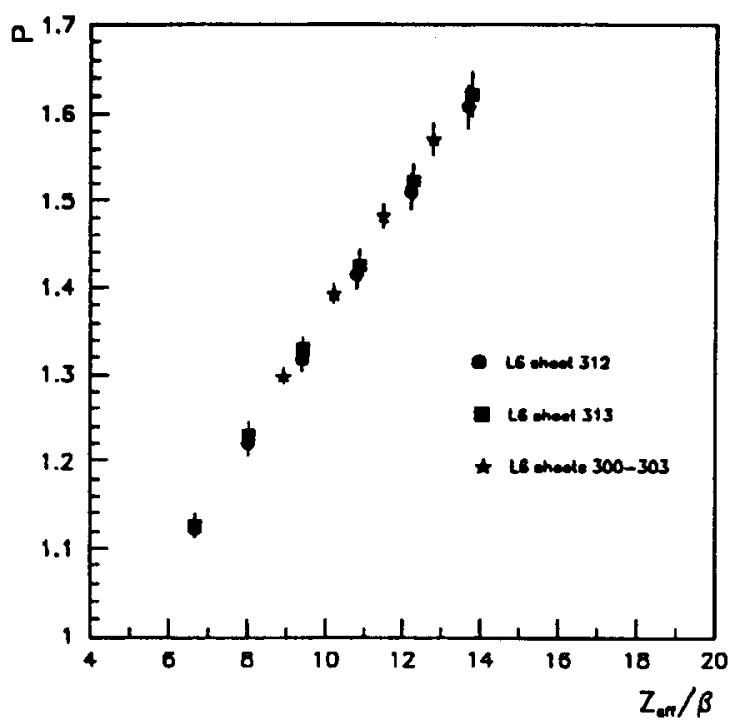

Fig.3. Mesured values of $p$ versus $Z_{e f f / B}$ for various sheets of CR39 of type $L 6$. Notice the reproducibility.

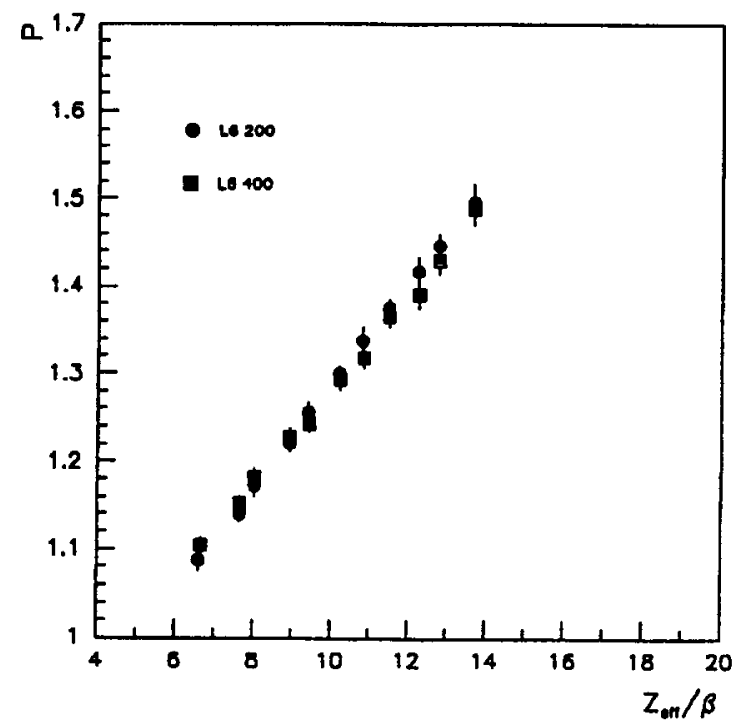

Fig.4. Measured values of $p$ versus $Z_{\text {eff }} / B$ for two sheets of CR39 type L6. Sheet L6 200 was one year old, while L6 400 was newly made. There is no indication of ageing.

\section{CONCLUSIONS}

The results indicate that for type L6,

(i) the quality of the surface and the transparency remain good after prolonged etching:

(ii) the threshold has been considerably reduced: the type EN3 has a threshold of $Z_{\text {eff }} / B=7.5$, while for $L 6$ it is at $Z_{\text {eff }} / B=5.5$;

(iii) the stability in sensitivity over a long period of time in the MACRO environment has been verified as well as the reproducibility among different sheets of a batch. 
The results prove that both EN3 and L6 types are adequate for the search for magnetic monopoles in MACRO (MACRO Collaboration, 1990). The relatively large difference in threshold between samples EN3 and L6 makes only a relatively minor effect on the B-threshold of the monopoles. We thank the staff of the Bevalac accelerator in Berkeley for their cooperation and our measuring staff for their untiring effort.

\section{REFERENCES}

Doke, T., Tawara, H., Hayashi, T., Ichinose, H., Kuwahara, K., Nakamura, S., Orito, S. and Ogura, K. (1988). CR39 plastic for massive magnetic monopole search. Nucl. Inst. and Meth., B34, 81-88 Fleischer, R. Price, P.B. and Walker, R.M. (1976). Nuclear Tracks in Solids. pg 18. California Press, Los Angeles.

MACRO Collaboration (1986). MACRO, A large area detector at the Gran Sasso Laboratory. Nuovo Cimento, 9C, 281 .

MACRO Collaboration (1988). The track etch detector of the MACRO experiment. 14th Int.Conf.Solid State Nuclear Track Detectors. Lahore, Pakistan.

MACRO Collaboration (1990).The track etch detector of the MACRO experiment at the Gran Sasso Laboratory. XXI ICRC, vol. HE 7.2 - 10, pp. 252. Adelaide, Australia. 